\title{
Analytical analysis of sensitivity of optical waveguide sensor
}

\author{
Alka Verma $^{1}$, Y. Prajapati ${ }^{1}$, S. Ayub ${ }^{1}$, J.P. Saini ${ }^{1}$, V. Singh ${ }^{2}$ \\ ${ }^{1 *}$ Department of Electronics and Communication Engineering, BIET, Jhansi, INDIA \\ ${ }^{2}$ Department of Physics, BHU Varanasi, INDIA \\ *Corresponding Author: e-mail: yogendrapra@gmail.com, Tel +91-9415909685
}

\begin{abstract}
In this article, we carried out analytical analysis of sensitivity and mode field of optical waveguide structure by use of effective index method. This structures as predicted have extended mode which could interact with the surrounding analyses in a much better way than the commonly used EWS.
\end{abstract}

Keywords: Sensor, Evanescent wave, Integrated fiber sensor, Nano-technology.

\section{Introduction}

Optical sensors utilize the modification of measurands to optical properties such as intensity, phase, frequency, and polarization of an input optical signal. Optical chemical sensors have immunity to electromagnetic interference, have no danger of ignition, and are compatible with fiber networks for use in remote spectroscopy and distributed sensing. Such kinds of sensor are useful for highly sensitive analysis and monitoring of hazardous environments and remote sensing (Brecht and Gauglitz, 1997). Demands for optical sensors have tremendously increased over the years due to issues concerning environmental pollution and other biohazards. In the recent years, optical sensors have attracted considerable attention, especially in the application of biochemical species detection. They have excellent advantages such as good compactness and robustness, immunity to electromagnetic interference, high sensitivity, shorter response time, low cost, and high compatibility with fiber optic networks (Passaro et al., 2007). However, optical fiber-based systems do not seem promising with respect to fabrication, efficiency and miniaturization. However, planar waveguide-based platforms employing evanescent wave sensing techniques have shown tremendous improvement (Burke et al., 2006) and evanescent wave sensors have proven to be highly sensitive (Brandenburg et al., 1995; Helmers et al., 1995; Pandraud et al., 2000). Thus, an optical sensor with good detection scheme, sensitivity and low cost is needed. The major contributions of this paper are as follows:

- Evanescent wave sensing (EWS) technique for ammonia (gaseous) detection utilizing a optical waveguide structure.

- Effective refractive index is a crucial parameter for analyzing the sensitivity;

The rest of this paper is organized as follows. Section 2 provides the principle and basic theory of optical sensors and the modal field and sensitivity, and its validation is presented. In sections 3, results and discussion of the optical sensors and compare it other fiber sensors. Finally, Section 4 concludes the paper.

\section{Theoretical Detail}

Evanescent waves are those waves which penetrate into the cladding or the region where light is reflected off the surface. In optical terminology, when total internal reflection occurs at an angle greater than the critical angle, the sinusoidal waves reflect off from an interface, and the waves which penetrate into, are called the evanescent waves (Qing et al., 1999). Fig.1 shows the evanescent field decaying exponentially into the cover region. The above mentioned mechanism is used in sensing, when the analytes or species to be detected are in contact or are made to interact with the evanescent field (which decay exponentially from the surface of the waveguide), forms the basis for evanescent wave sensing. The operating wavelength is selected such that the 
analytes show peak absorption, so that there is a change at the output either in the intensity or power or any other parameter, when measured using a detector.

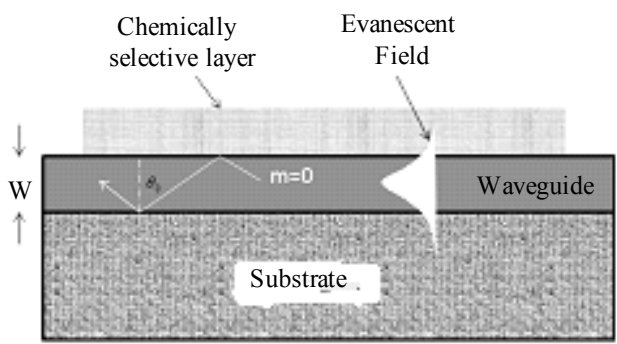

Fig. 1: Single mode waveguide with Evanescent wave Penetration.

Generally, effective refractive index $\mathrm{N}_{\text {eff }}$ of a guided mode is considered to be most crucial physical parameter. It is because in this paper for optical sensor, a chemically selective layer or adlayer is deposited on the waveguide surface to bind the analyte. The evanescent field senses the changes in the refractive index caused by the layer, thus inducing an effective refractive index change (Lukosz et al., 1995; Campbell et al., 1999). The difficulty faced by most evanescent wave sensor architectures lies in the lack of strong interaction between the analytes (target species) and the evanescent field. This is primarily due to the use of strongly confined waveguide designs (Whaley et al., 2007). So we utilize the concept of small optical mode area structure in core, where a nanoscale core region is used, which results in a large and distended mode. These structures have a very small percentage (usually less than 1\%) of the optical mode confined in the guiding material. These large modes are sufficient to provide a strong interaction with the analytes, as the field is pushed out of the core region, enhancing the field interaction with the analytes. All the upper and lower cladding layer are air, while core is dielectric or depending upon application. In this paper used planer symmetric waveguide shown in fig.2.

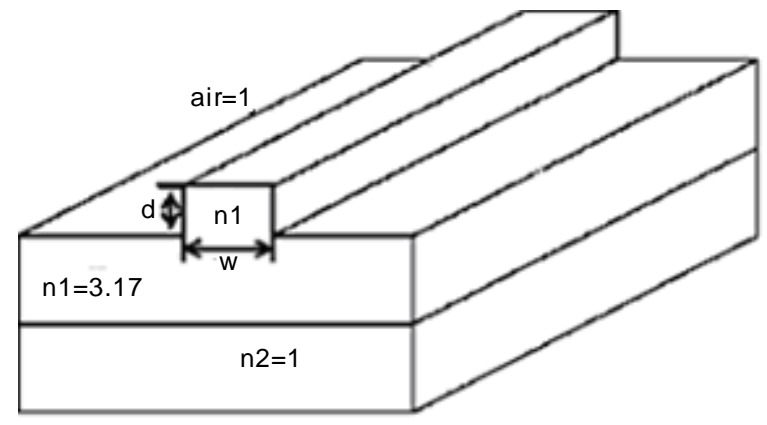

Fig. 2: Planer waveguide structure

\subsection{Brief Review of Effective Index for a Symmetric Planar Waveguide structure:}

From (Koshiba, 1973), we understand that "a waveguide mode is a transverse field pattern whose amplitude and polarization profile remains constant along the longitudinal direction". The electric and magnetic fields of a mode can be written as

$$
\begin{aligned}
& E(r, t)=E_{m}(x, y) e^{(i \beta z-i \omega t)} \\
& H(r, t)=H_{m}(x, y) e^{(i \beta z-i \omega t)}
\end{aligned}
$$

Where ' $\mathrm{m}$ ' is the mode index, $E_{m}(x, y)$ and $H_{m}(x, y)$ are the mode field patterns and $\beta$ is the propagation constant of the mode. Using the basic Maxwell Equations, we obtain the TE and TM modes respectively.

$$
\begin{aligned}
& \frac{\partial^{2} E_{y}}{\partial x^{2}}+\left(k_{0}^{2}-\beta^{2}\right) E_{y}=0 \quad(\text { for TE modes }) \\
& \frac{\partial^{2} H_{y}}{\partial x^{2}}+\left(k_{0}^{2}-\beta^{2}\right) H_{y}=\frac{1}{\varepsilon} \frac{\partial \varepsilon}{\partial x} \frac{\partial H_{y}}{\partial x} \quad(\text { for TM modes })
\end{aligned}
$$

where $k_{0}=\frac{\omega^{2}}{c^{2}} n^{2}(x)$

A guided mode is one in which the power is retained inside the core as the wave, is perfectly reflected off both the interfaces. This mode can exist only if it satisfies a transverse resonance condition, such that the reflected wave has constructive interference with itself. The transverse component (x-component) of the wave vector inside the core is $\mathrm{h}_{1}=\mathrm{k}_{01} \cos \theta$, where $\theta$ is the angle of incidence, $k_{0 i}=2 \pi n i / \lambda, i=1,2,3$ and the longitudinal component (z-component) $\beta=\mathrm{k}_{01} \sin \theta$. Similarly, the transverse 
components for the substrate and cover regions can be defined as $\mathrm{h}_{2}=\mathrm{k}_{02} \cos \theta$, and $\mathrm{h}_{3}=\mathrm{k}_{03} \sin \theta$, where $\mathrm{k}_{01}, \mathrm{k}_{02}$ and $\mathrm{k}_{03}$ are the propagation constants in the respective regions. Applying boundary condition at $n_{1} / n_{2}$ and $n_{1} / n_{3}$ the interface, and using the above equation (2), we obtain the eigen value equation for TE and TM mode respectively.

$$
\begin{aligned}
& \tan \left(\frac{h_{1} d}{2}-\frac{m \pi}{2}\right)=\frac{\sqrt{V^{2}-h_{1}^{2} d^{2}}}{h_{1} d}, m=0,1,2 \ldots .(\text { for TE } \bmod e) \\
& \tan \left(\frac{h_{1} d}{2}-\frac{m \pi}{2}\right)=\frac{n_{1}^{2}}{n_{2}^{2}} \frac{\sqrt{V^{2}-h_{1}^{2} d^{2}}}{h_{1} d}, m=0,1,2 \ldots .(\text { for TM mode })
\end{aligned}
$$

The equations above mentioned are solved using Matlab, graphically by plotting left hand side and right hand side, as a function of (h1d). The solutions give the value of $\beta$, and hence the effective index, Neff, could be calculated from the relation Neff $=\lambda \beta / 2 \pi$.

2.2 General Expression for sensitivity: For a planer optical waveguide consisting of charge free, homogeneous, isotropic media, sensitivity is defined "as the rate of change of the modal effective permittivity, relative to the cover permittivity," where $\varepsilon=\mathrm{n}_{2}$. However, in case of optical detection, we are more interested in the phase of a light wave than dielectric constant. Hence the sensor sensitivity is redefined as the rate of change of effective index relative to its cover index change, in case of homogeneous sensing and in case of surface sensing, it's relative to the change in the film thickness. Using the variational theorem as mentioned in (Dell'Olio et al., 2007), for dielectric waveguides, we obtain the equation of sensitivity for quasi TE and quasi TM modes as the

following

$$
S_{T E}=\frac{1}{N} \frac{Q+k_{0} \omega n_{2}^{3} A_{i}^{T E}\left(n_{1} a_{2} \sqrt{a_{2}+n_{2} a_{1} \sqrt{a_{2}}}\right)}{k_{0} \omega n_{2}^{3} \sqrt{a_{2}}\left(n_{1} a_{2}+n_{2} a_{1}\right)+2 a_{1}^{2} a_{2}^{2}\left(a_{1}+a_{2}\right)}
$$

$$
S_{T M}=\frac{1}{N}\left[A_{i}^{T M}+\frac{2 a_{1}\left(A_{2}^{T M}-A_{1}^{T M}\right)}{\left(2+k_{0} \omega \sqrt{a_{2}}\right)\left(a_{1}+a_{2}\right)}\right]
$$

where

$$
\begin{aligned}
& Q=4 a_{1} a_{2} A_{2}^{T E} n_{1}^{2}-4 a_{1} a_{2} A_{1}^{T E} n_{2}^{2}+2 a_{2} A_{1}^{T E} a_{1}^{2} a_{2}^{2}+2 a_{1} A_{2}^{T E} a_{1}^{2} a_{2}^{2} \\
& a_{1}=n_{1}^{2}-N^{2} \text { and } a_{2}=N^{2}-n_{2}^{2}
\end{aligned}
$$$$
A_{i}^{T E}=\frac{n_{c}}{\sqrt{c_{i}\left(1+\frac{c_{i}}{f_{i}}\right)\left(h_{i} k_{0}+\frac{1}{\sqrt{c_{i}}}+\frac{1}{s_{i}}\right)}} i=1,2
$$$$
A_{i}^{T M}=\frac{\left(2 c_{i}+n_{c}^{2}\right)\left(\frac{c_{i} n_{f}^{4}+f_{i} n_{c}^{4}}{f_{i} n_{c}^{4}}\right)^{-1} \frac{1}{n_{c}^{3} \sqrt{c_{i}}}}{\left[\frac{h_{i} k_{0}}{n_{f}^{2}}+\frac{\left(c_{i}+f_{i}\right) n_{c}^{2}}{\left(c_{i} n_{f}^{4}+f_{i} n_{c}^{4}\right) \sqrt{c_{i}}}+\frac{\left(s_{i}+f_{i}\right) n_{s}^{2}}{\left(s_{i} n_{f}^{4}+f_{i} n_{s}^{4}\right) \sqrt{s_{i}}}\right]} i=1,2
$$

$c_{i}=n_{i}^{2}-n_{c}^{2} \quad i=1,2$

$s_{i}=n_{i}^{2}-n_{s}^{2} \quad i=1,2$

$f_{i}=n_{f}^{2}-n_{i}^{2} \quad i=1,2$

where $\mathrm{k}_{0}$ is the vacuum wave number or propagation constant, $\mathrm{n}_{1}$ is the effective index obtained for the structure with height $\mathrm{h}_{1}$ and $\mathrm{n}_{2}$ is the effective index obtained for the structure with height $\mathrm{h}_{2}$, (as shown in the Figure2).

\section{Results and Discussions}

In order to get sensitivity of the above proposed waveguide, we have to solve the equation (3) numerically. For this purpose we have chosen the following parameters: Region 1 consists of a core index $n_{1}=3.16$ and $n_{2}=n_{3}=1$, with a width of $100 \mathrm{~nm}$, while regions 2 and 3, have the similar index distribution, but the width is $50 \mathrm{~nm}$ each using wavelength $1.55 \mu \mathrm{m}$. For the structure proposed here, $\beta_{2}=\beta_{3}$. After obtaining the three effective indices, we treat the structure with $\mathrm{n}_{1}=$ Neff1 and $\mathrm{n}_{2}=\mathrm{n}_{3}=$ Neff 2 or Neff3.Now, we apply the above procedure again to calculate the propagation constant for the final structure, having a ydependence on the y-component for the TE like mode or the electric field Ey and the magnetic field Hy for TM mode in figure3. Firstly, the three regions are analyzed in the x-direction (vertical), the corresponding effective indices are obtained and those 
effective indices are considered to get a final structure in the y-direction (horizontal), with a width of $3 \mu \mathrm{m}$. This final structure is again a three slab symmetric waveguide as mentioned before, but for this final structure, to obtain a TE- like mode, we consider the TM field of the vertical structure; and for TM-like mode, it's the TE field of the initial vertical structure. The Neff obtained from the analytical calculations are 1.6856 and 1.00122 for TE mode and TM mode respectively. In the case of homogeneous

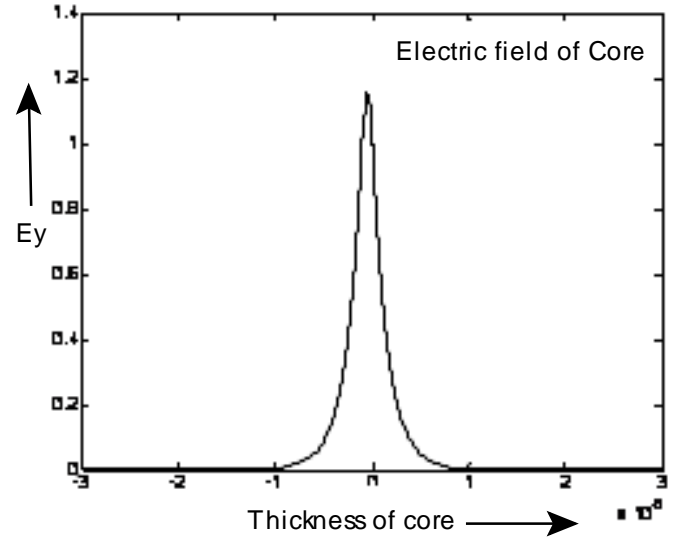

(a)

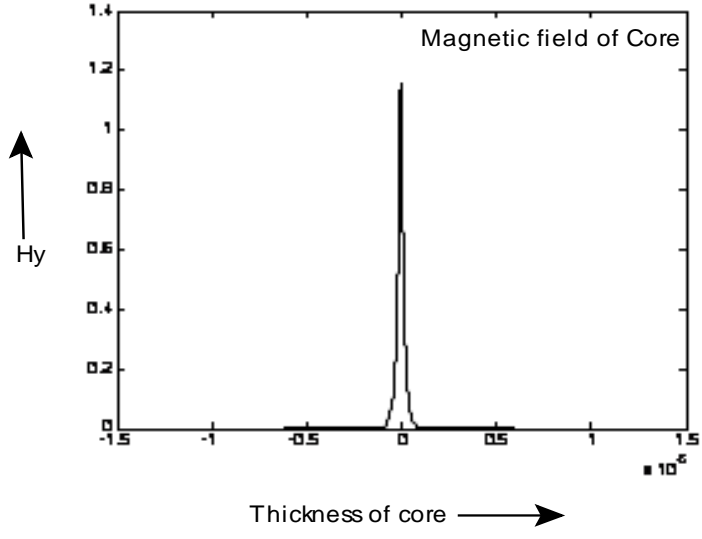

(b)

Fig.3. Field distribution (a) Electric field (b) Magnetic field

sensing, we obtained the sensitivity of TE modes have 0.8749 and TM modes have 0.3882 . This result shows that TE modes more sensitivity to TM modes.

\section{Conclusions}

The purpose of this paper is to introduce small optical mode area structure in core based on Evanescent wave sensing in Integrated Optics (IO) sensors. This structures as predicted have extended mode which could interact with the surrounding analyses in a much better way than the commonly used EWS. The shortcomings of EWS are overcome by the introduction of the small optical mode area structure technique. The modal analysis of small optical mode area structure in core structures used in both gaseous sensing is studied.

\section{References}

Brecht A. and Gauglitz G., 1997. Recent developments in optical transducers for chemical or biochemical applications, Sens. Actuators Vol. B38-39, pp. 1-7.

Brandenburg A., Rainer Edelhauser, Tobias Werner, Huarui He, and Otto S. Wolfbeis, 1995. Ammonia Detection via Integrated Optical Evanescent Wave Sensors, Mikrochim. Acta., Vol. 121, pp. 95-105.

Campbell D.P., Moore J.L., Cobb J.M., Hartman N.F., Schneider B.H. and Venugopal M.G., 1999. Optical system-on-a-chip for chemical and biochemical sensing: The chemistry, Chemical, Biochemical, and Environmental Fibre Sensors X, Vol. 3540, pp. 153-161.

Conor S. Burke, Ondrej Stranik, Helen M. McEvoy, and Brian D. MacCraith, "Planar Optical Sensors and Evanescent Wave Effects," Optical Chemical Sensors, pp. 193-215, 2006.

De-Kui Qing and Ichirou Yamaguchi "Analysis of the sensitivity of optical waveguide chemical sensors for TM modes by the group-index method" Vol. 16, No. 9/September 1999/J. Opt. Soc. Am. B..

Dell'Olio F., Passaro V. M. N. and De Leonardis F., 2007. Sensitivity analysis of rib waveguides for integrated optical sensors," 1-4244-1245-5/07, IEEE.

Hakon Helmers, Pierre Greco, Rolf Rustad, Rochdi Kherrat, Gerard Bouvier, and Pierre Benech, "Performance of a compact, hybrid optical evanescent-wave sensor for chemical and biological applications," Applied Optics, vol. 35, no. 4,Feb 1996.

Koshiba, M. 1973. Optical waveguide analysis, New York, McGraw-Hill.

Lukosz W., 1995. Integrated optical chemical and direct biochemical sensors, Sensors and Actuators B, vol. 29, pp. 37-50.

Pandraud G., T. M. Koster, C. Gui, M. Dijkstra, A. van den Berg, and P. V.Lambeck, "Evanescent wave sensing: new features for detection in small volumes," Sensors and Actuators, vol. 85, pp. 158-162, 2000.

Passaro V. M. N., Dell'Olio F., Casamassima B., and De Leonardis F., 2007. Guided wave optical biosensors, Sensors, Vol. 7, pp. 508-536. 
Whaley R. D. Jr., Kwakernaak M. H., Khalfin V. B., Lipp S. A., Chan W. K., An H, and Abeles J. H., 2007. Observation of low optical overlap mode propagation in nanoscale indium phosphide membrane waveguides, Appl. Phys. Lett., Vol. 90, pp. 011114-1-011114-3.

\section{Biographical notes}

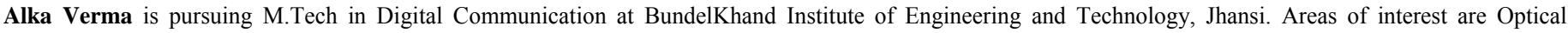
Communication, optical waveguide sensor and Optoelectronics.

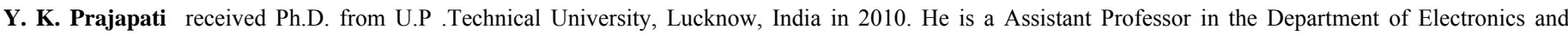

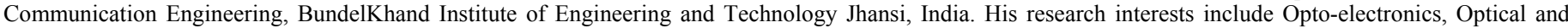

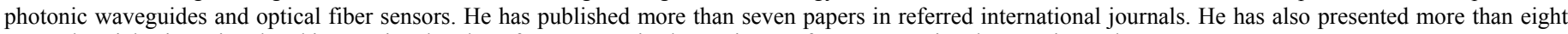
research articles in national and international and conferences. He is also reviewer of two International repute journals.

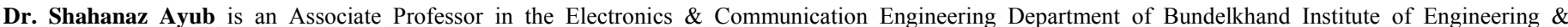

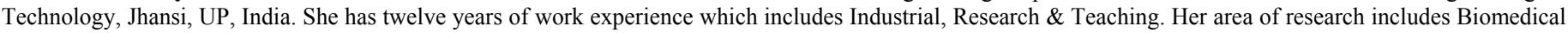

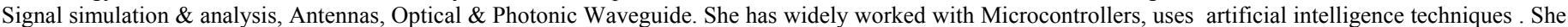
has published thirteen research papers in International journals, presented papers in twenty three national \& international conferences.

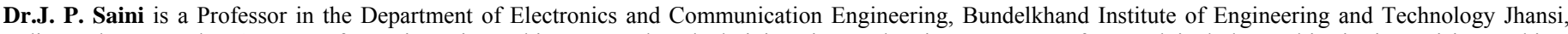

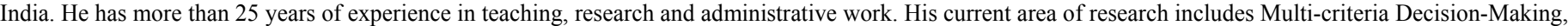

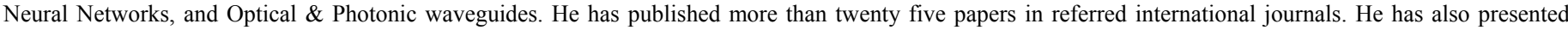

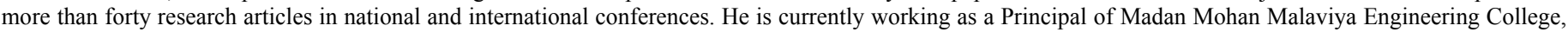
Gorakhpur, India on deputation.

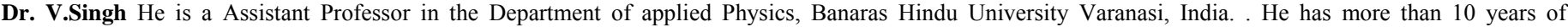

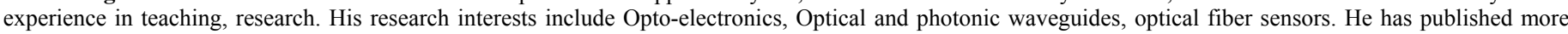

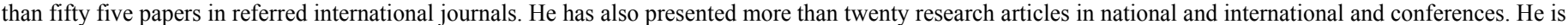
currently dealing with few projects sponsored by government of India.

Received January 2011

Accepted March 2011

Final acceptance in revised form April 2011 